\title{
Prozess-Benchmarking in der österreichischen Trinkwasserwirtschaft
}

\section{Process benchmarking in the Austrian drinking-water industry}

\author{
von J. Kölbl'1 , H. Theuretzbacher-Fritz ${ }^{1}$, E. Mayr ${ }^{2}$, R. Neunteufel ${ }^{2}$ und R. Perfler ${ }^{2}$
}

\begin{abstract}
Kurzfassung/Summary
Mit der Zielsetzung eines qualitativ hochwertigen Leistungsvergleiches einzelner Prozesse unter Berücksichtigung sowohl wirtschaftlicher wie auch qualitativer Aspekte wurde im Zeitraum von März 2007 bis März 2008 Prozess-Benchmarking erfolgreich in die österreichische Trinkwasserwirtschaft eingeführt. Aufbauend auf dem ganzheitlichen Zugang des Unternehmensbenchmarking wurden unter der Trägerschaft der Österreichischen Vereinigung für das Gas- und Wasserfach (ÖVGW) sechs ausgewählte Prozesse zu den drei Themenbereichen Wasserverkauf, Leitungsbau sowie Netzbetrieb und Instandhaltung auf freiwilliger Basis untersucht. Dieser Beitrag liefert die wichtigsten Eckdaten dieses Projekts und beschreibt ausgewählte methodische und fachliche Ergebnisse.
\end{abstract}

With the aim of enabling high-quality performance comparisons among individual processes under both economic and qualitative aspects, process benchmarking was successfully introduced into the Austrian drinking-water industry between March 2007 and March 2008. Six selected processes regarding three fields - water sale, pipeline construction as well as system operation and maintenance - were studied on a voluntary basis utilising the holistic approach of company benchmarking, under the auspices of Österreichische Vereinigung für das Gas- und Wasserfach (Austrian Association for Gas and Water-OVGW). This article presents the main key data of this project and describes selected methodical and technical results.

\section{Einleitung}

In vielen europäischen und außereuropäischen Ländern wurden in den letzten Jahren im Bereich der Wasserversorgung bzw. Abwasserentsorgung verschiedene Benchmarkingmodelle vor dem Hintergrund verschiedener Motivationen und unter unterschiedlichen Randbedingungen entwickelt. Neben verschiedenen Ansätzen zum Unternehmens-Benchmarking, wo das gesamte Unternehmen auf Basis von teilweise hoch aggregierten Kennzahlen zu den verschiedenen Aufgabengebieten untersucht wird, geht auch international der Trend in letzter Zeit vermehrt zu ergänzenden, tiefer gehenden Untersuchungen einzelner Arbeitsprozesse, was man als Prozess-Benchmarking bezeichnet.

Die Österreichische Vereinigung für das Gas- und Wasserfach (ÖVGW) als Projektträger organisiert seit einigen Jahren ein Benchmarking für die österreichische Trinkwasserwirtschaft (siehe Abbildung 1), welches in Kooperation mit der Technischen Universität Graz und der BOKU Wien durchgeführt wird. Nach zwei Projekten mit Unternehmens-Benchmarking im Zeitraum von 2003 bis 2006 wurde 2007 mit Prozess-Benchmarking begonnen. Erfahrungen aus dem Unternehmens-Benchmarking (den Stufen A und B des ÖVGW Benchmarking) zeigen, dass sich das Unternehmens-Benchmarking gut eignet, unterschiedliche Wasserversorgungsunternehmen hinsichtlich ihrer Stärken und Schwächen zu untersuchen und, gruppiert nach verschiedenen Kriterien, auch miteinander $\mathrm{zu}$ vergleichen. Zur
Ableitung von konkreten Verbesserungsmaßnahmen sind aber oft vertiefende Analysen erforderlich. Auch internationale Erfahrungen zeigen, dass wirkliche Effizienzsteigerungen ohne eine Betrachtung einzelner Prozesse nur schwer möglich sind (z. B. Otillinger, 2004).

\section{Zielsetzung}

DieZielsetzungdesösterreichischen Prozess-Benchmarking Projektes ist eine starke Fokussierung auf konkrete Verbesserungspotentiale in den einzelnen Prozessschritten. Neben den Prozesskosten ist auch der Vergleich der Qualität in der Prozessdurchführung ein wichtiges Ziel. Eine strukturierte Datenerhebung stellt die Basis für eine qualitative und quantitative Erfassung der einzelnen Prozessschritte dar.

Eine Prämisse war die Berücksichtigung der jeweiligen Rahmenbedingungen und Strukturen der teilnehmenden Wasserversorgungsunternehmen sowie die in Österreich typischen Verhältnisse hinsichtlich z. B. Kostenrechnung oder Ausschreibungsmodalitäten.

Im Aufbau des Prozess-Benchmarking-Systems war neben dem Einfließen von internationalen Erfahrungen in diesem Bereich auch das Zurückgreifen auf grundsätzliche Erfahrungen aus dem Abwasserprojekt (Benchmarking in der Siedlungswasserwirtschaft, BOKU Wien) von Bedeutung.

\section{Methodik}

Im Zuge des Prozess-Benchmarking 2007/08 wurden insgesamt sechs Prozessvergleiche $\mathrm{zu}$ den drei Themenschwerpunkten Was- 
serverkauf, Leitungsbau sowie Netzbetrieb und Instandhaltung durchgeführt (siehe Tabelle 1). Die Auswahl der untersuchten Prozesse erfolgte in einer Arbeitsgruppe mit VertreterInnen der ÖVGW, von fünf interessierten Wasserversorgungsunternehmen und des Projektteams. Diese Arbeitsgruppe begleitete das gesamte Projekt und war in die Erarbeitung der einzelnen Prozesse eingebunden. Dabei wurden besonders die vorgeschlagenen unterschiedlichen methodischen Möglichkeiten zur Datenerfassung ausgearbeitet bzw. diese Methoden aus Sicht der Praxis beurteilt. Nach Ausarbeitung der Erhebungsunterlagen zu den einzelnen Prozessen und Praxistests durch Mitglieder der Arbeitsgruppe erfolgte die Datenerhebung durch die teilnehmenden Wasserwerke und anschließend die Auswertung durch das Projektteam. Zentraler Teil des Projektes waren Workshops zur Ursachenanalyse, wo die Ergebnisse aus dem Prozessvergleich präsentiert und im Teilnehmerkreis hinsichtlich der methodischen und fachlichen Erkenntnisse diskutiert wurden. Den Teilnehmern wurden weiters vertrauliche Individualberichte für die weitere unternehmensinterne Bearbeitung zur Verfügung gestellt.

Die Teilnahme am Projekt war freiwillig und es war möglich auch an einzelnen Prozessen teilzunehmen.

\section{Ergebnisse}

Da das ÖVGW Prozess-Benchmarking Projekt in den Jahren 2007/08 zum ersten Mal durchgeführt wurde, waren neben fachlichen Ergebnissen v.a. auch methodische Erkenntnisse zu erwarten. Im Folgenden sind einige ausgewählte Projektergebnisse beschrieben.

\subsection{Themenbereich Wasser- verkauf}

Im Themenbereich Wasserverkauf wurden mit der Zählerablesung und Verbrauchsabrechnung sowie

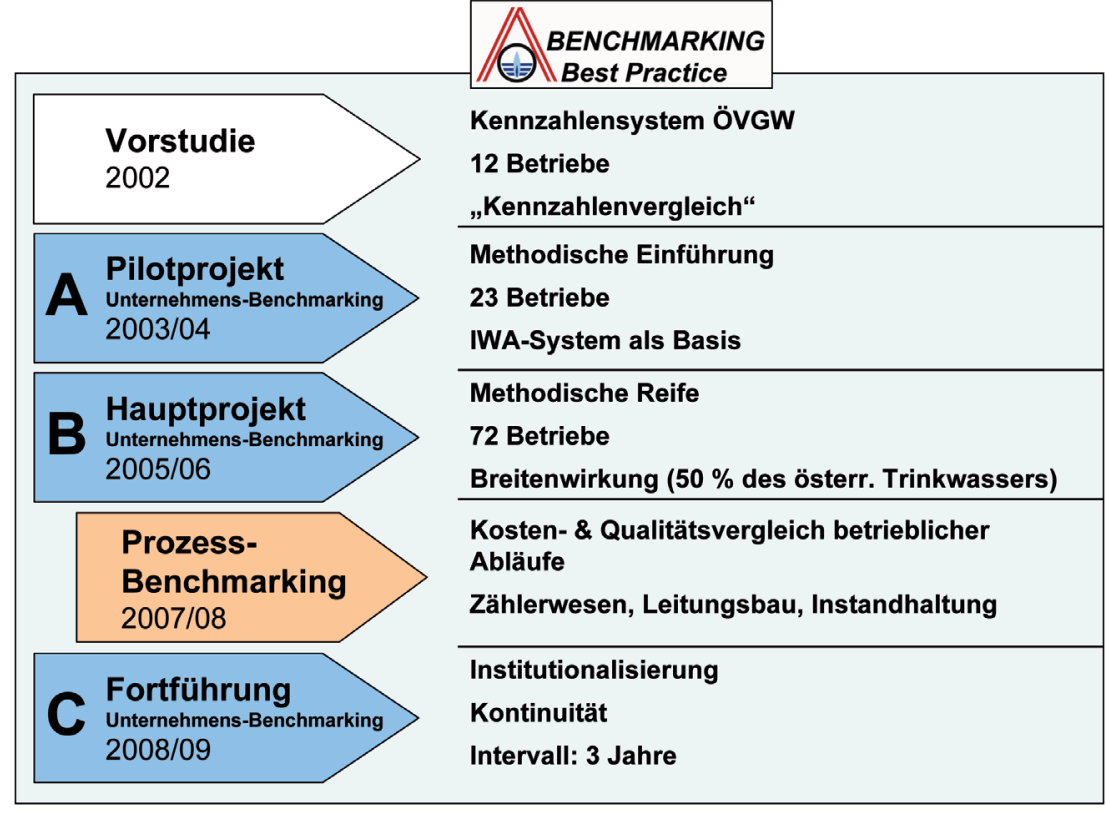

Abb. 1: ÖVGW Benchmarking Strategie

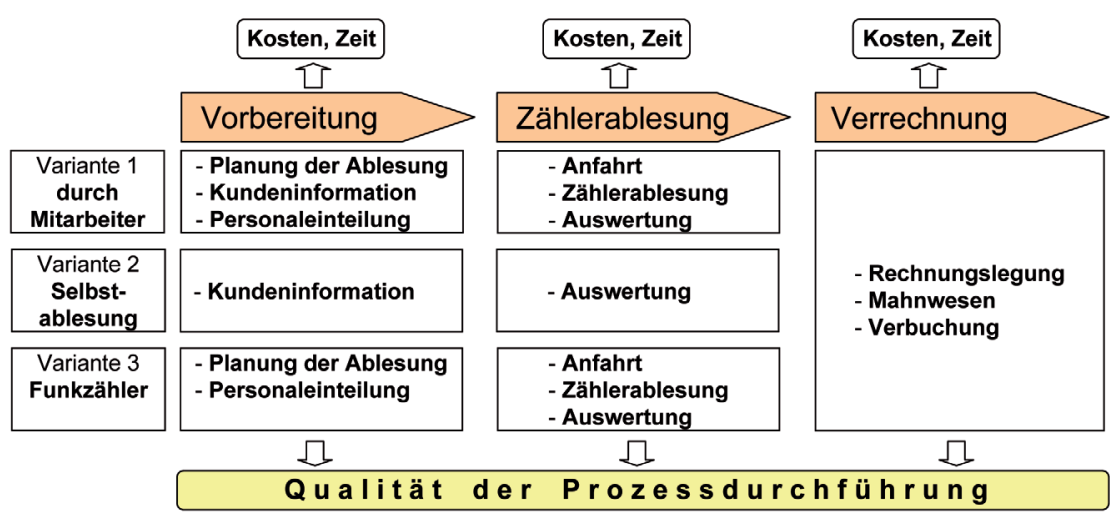

Abb. 2: Prozessstruktur Zählerablesung und Verbrauchsabrechnung (Kölbl et al., 2008)

\begin{tabular}{|c|c|c|}
\hline \multirow{2}{*}{ Wasserverkauf } & $\begin{array}{l}\text { Zählerablesung und } \\
\text { Verbrauchsabrechnung }\end{array}$ & 15 Teilnehmer \\
\hline & Zählertausch & 16 Teilnehmer \\
\hline \multirow{3}{*}{ Leitungsbau } & $\begin{array}{l}\text { Neuerrichtung von Haupt- } \\
\text { und Versorgungsleitungen }\end{array}$ & $\begin{array}{l}6 \text { Teilnehmer mit } \\
6 \text { Leitungsbauprojekten }\end{array}$ \\
\hline & $\begin{array}{l}\text { Rehabilitation von Haupt- } \\
\text { und Versorgungsleitungen }\end{array}$ & $\begin{array}{l}10 \text { Teilnehmer mit } \\
11 \text { Leitungsbauprojekten }\end{array}$ \\
\hline & $\begin{array}{l}\text { Erneuerung von Haus- } \\
\text { anschlussleitungen }\end{array}$ & $\begin{array}{l}10 \text { Teilnehmer mit } \\
11 \text { Leitungsbauprojekten }\end{array}$ \\
\hline $\begin{array}{l}\text { Netzbetrieb und } \\
\text { Instandhaltung }\end{array}$ & Wasserverlustmanagement & 11 Teilnehmer \\
\hline
\end{tabular}




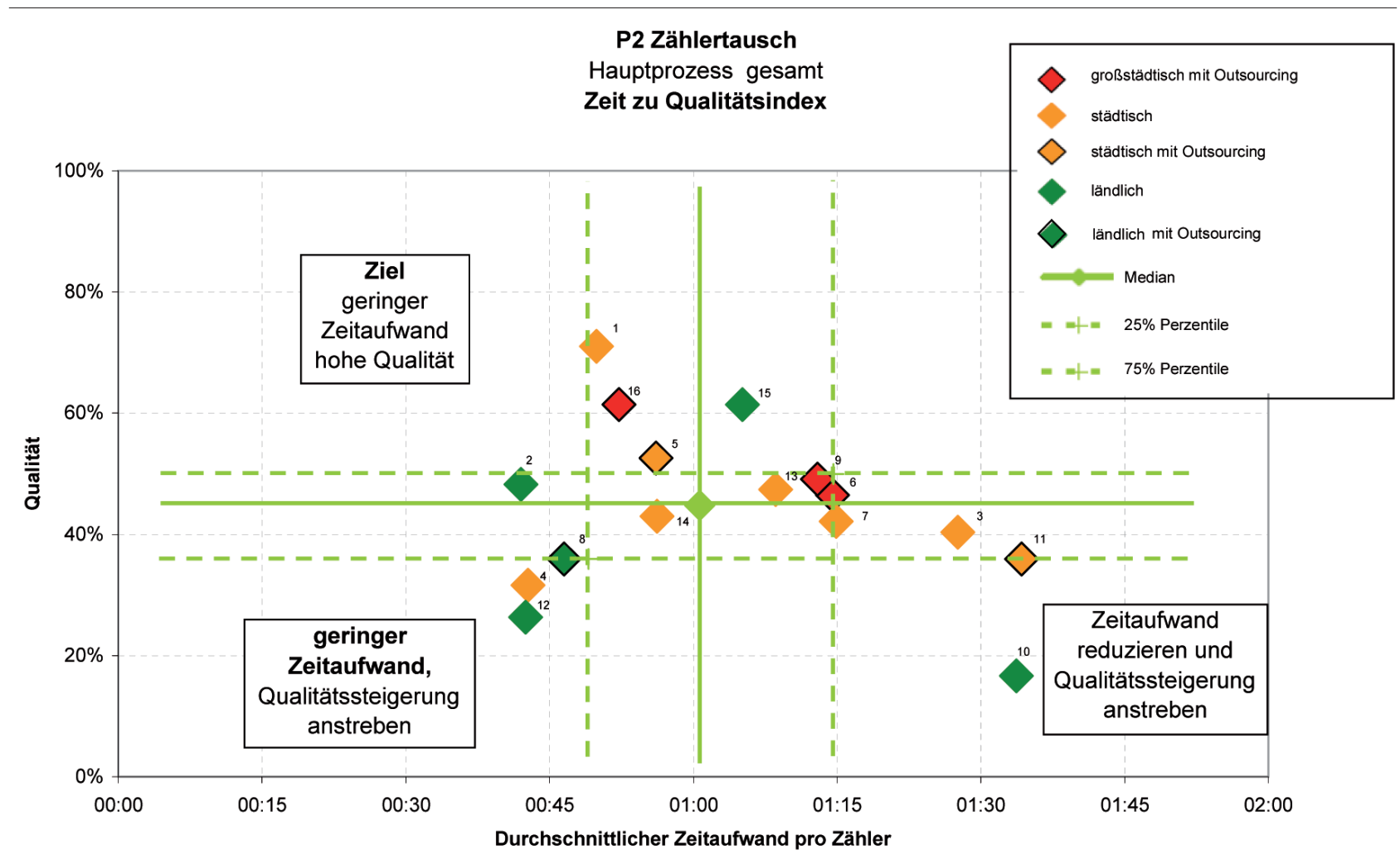

Abb. 3: Zeit-Qualitäts-Diagramm für den Prozess Zählertausch

Tab. 2: Rahmenbedingungen der verglichenen Projekte im Prozess „Rehabilitation von Haupt- und Versorgungsleitungen"

\begin{tabular}{|c|c|c|c|c|c|c|c|c|c|}
\hline $\begin{array}{l}\text { WVU } \\
\text { Nr. }\end{array}$ & $\begin{array}{c}\text { Aus- } \\
\text { schreib- } \\
\text { ung }\end{array}$ & Planung & $\begin{array}{l}\text { Erd- } \\
\text { arbeiten }\end{array}$ & $\begin{array}{l}\text { Installa- } \\
\text { tion }\end{array}$ & Material / DN & Bauweise & Oberfl. & $\begin{array}{l}\text { Mit- } \\
\text { verl. }\end{array}$ & $\begin{array}{l}\text { Länge } \\
\text { (m) }\end{array}$ \\
\hline 1 & JBV & intern & \multirow{11}{*}{ extern } & extern & Pe-DA110-160 & Künette & \multirow{11}{*}{ vers. } & nein & 1203 \\
\hline 2 & EP & extern & & intern & $\begin{array}{l}\text { GGG-300 und } \\
\text { Pe-DA110 }\end{array}$ & Künette & & nein & 1430 \\
\hline 3 & EP & intern & & intern & Pe-DA140-63 & Künette & & ja & 915 \\
\hline 4 & EP & intern & & extern & GGG-125-100 & Künette & & nein & 210 \\
\hline 5 & EP & intern & & extern & GG-375 & $\begin{array}{l}\text { Schlauch- } \\
\text { relining }\end{array}$ & & ja & 850 \\
\hline 8 & JBV & intern & & intern & Pe-DA160 & Künette & & ja & 522 \\
\hline 10 & EP & intern & & intern & Pe-DA110-90 & $\begin{array}{l}\text { Rohrauswechs- } \\
\text { lungsverfahren / } \\
\text { sonstige }\end{array}$ & & nein & 380 \\
\hline 11 & JBV & intern & & intern & St-100 & Künette & & ja & 300 \\
\hline $12 \mathrm{a}$ & EP & $\begin{array}{l}\text { outsourcing- } \\
\text { inhouse }\end{array}$ & & intern & Pe-DA110 & Künette & & nein & 143 \\
\hline $12 b$ & EP & intern & & extern & Pe-DA110 & Relining & & nein & 259 \\
\hline 14 & EP & extern & & intern & ST-200-150 & Künette & & nein & 1124 \\
\hline
\end{tabular}


dem Zählertausch insgesamt zwei Prozesse untersucht.

Das Ziel für das Benchmarken des Prozesses Zählerablesung und Verbrauchsabrechnung ist das Analysieren aller für die Prozessdurchführung erforderlichen Tätigkeiten hinsichtlich Kosten und Qualität. Eine besondere Herausforderung für den Prozessvergleich stellen die drei möglichen Varianten der Prozessabwicklung dar: Ablesung durch MitarbeiterInnen des Wasserversorgungsunternehmens (Variante 1), Selbstablesung durch die Kunden (Variante 2) und Ablesung mittels Funkzähler (Variante 3). Die Prozessstruktur mit den drei möglichen Varianten ist in Abbildung 2 ersichtlich. Über den Vergleich der verschiedenen Varianten ist es für jeden Teilnehmer möglich, die für die jeweiligen Rahmenbedingungen günstigste Variante bzw. Variantenkombination abzuleiten (Kölbl et al., 2008).

Das Aufzeigen einer absoluten Best Practice bei Zählerablesung und Verbrauchsabrechnung ist aufgrund der vielen Besonderheiten der unterschiedlichen Versorgungssysteme der 15 Teilnehmer nicht möglich. Vielmehr kann man von mehreren Best Practices sprechen, die zu einer effizienten und erfolgreichen Durchführung des Prozesses führen. Betreffend den effizienten Einsatz der drei unterschiedlichen Varianten konnte erkannt werden, dass ein Varianten-Mix durchaus von Vorteil sein kann. Wie bereits oben beschrieben, wurde aber auch erkannt, dass Betriebe, die nur eine Variante verwenden, mit einer routinierten Durchführung ein hohes Maß an Effizienz erreichen können. Das gilt für alle drei möglichen Varianten. Die Gesamtkosten für die Prozessdurchführung liegen je nach Variante und Rahmenbedingungen bei $50 \%$ des TeilnehmerInnenfeldes zwischen $€ 6$ und $€ 9$ je Zählerablesung (inkl. Abrechnung). Jeweils $25 \%$ des TeilnehmerInnenfeldes liegen darüber oder darunter.

Die oft vertretene Ansicht, dass die Selbstablesung durch den Kunden (z. B. mittels Ablesekarte oder Bekanntgabe via Internet) kostengünstiger als die Ablesung durch Mitarbeiter des Wasserversorgungsunternehmens sei, konnte anhand der Vergleichsergebnisse nicht eindeutig bestätigt werden. Mehrere Wasserversorgungsunternehmen begründeten dies damit, dass der Aufwand für die Ablesung jener Kundenzähler, von denen keine Rückmeldung kommt, überdurchschnittlich hoch ist. Einen Kostenvorteil gegenüber anderen Varianten konnten nur jene Wasserversorgungsunternehmen erzielen, die auf eine Ablesung jener Kundenzähler, von denen keine Rückmeldung kommt, verzichten und die Verbrauchswerte schätzen.

Was den Einsatz von Funkzählern betrifft hat sich gezeigt, dass einige Unternehmen sehr positive Erfahrungen mit dem Einsatz von Funkzählern gemacht haben. Wichtig ist dabei aber der Einsatz der neuesten Funkzählergeneration. Aus wirtschaftlicher Sicht wird der Vorteil einer effizienteren Ablesung durch die erhöhten Investitionskosten oftmals wieder aufgebraucht. Während einige Unternehmen ganze Ortschaften oder Städte flächendeckend mit Funkzählern ausstatten, setzen andere Wasserversorger auf den selektiven Einsatz von Funkzählern (z. B. Zähler in Schächten, Zweitwohnsitze, städtischer Bereich wenn durch geringe Anwesenheit der KundInnen der Zugang zu den Wasserzählern nicht gewährleistet ist). Grundsätzlich haben Funkzähler Vorteile betreffend Arbeitssicherheit, Kundenfreundlichkeit und beim Wasserverlustmanagement.

Auch beim zweiten Prozess dieses Themenbereiches, dem Zählertausch, musste zwischen verschieden Varianten differen- ziert werden: Tausch von „Normalzählern“ (Variante 1), Tausch von Funkzählern (Variante 2) sowie Ersteinbau von Funkzählern (Variante 3).

Abbildung 3 zeigt eine Übersicht der Arbeitszeiten für den Zählertausch. Dabei ist zu beachten, dass unterschiedliche Varianten gewichtet nach dem Anteil der jeweiligen Variante berücksichtigt werden. Des Weiteren sind jene TeilnehmerInnen gesondert gekennzeichnet, die Teile der Prozessabwicklung an Fremdfirmen oder andere Abteilungen innerhalb eines Konzerns ausgelagert haben, wobei die outgesourcten Leistungen in der Grafik nicht berücksichtigt sind.

Im Vergleich der Subprozesse Vorbereitung, Zählertausch und Nachbetreuung wird der überwiegende Teil der Zeitanteile vom Subprozess Zählertausch, mit den Teilaufgaben Anfahrt und Ein-/ Ausbau der Zähler, verbraucht. Die größten Kostenanteile werden überwiegend von den Beschaffungskosten für die Tauschzähler respektive für die Eichung sowie den Kostenanteilen für die Anfahrt und den Ein-/Ausbau der Zähler verursacht. Der Vergleich der Tauschzählerkosten und der Kosten für Eichung zeigte keine nennenswerten Unterschiede.

\subsection{Themenbereich Leitungsbau}

Kann bei Arbeitsabläufen zum Rohrnetzbau eine Qualitäts- und Kostenoptimierung erreicht werden, wirkt sich das überdurchschnittlich stark auf die langfristige Leistungsfähigkeit des Wasserversorgungsunternehmens aus, zumal das Rohrnetz mit ca. 50 bis $70 \%$ einen beträchtlichen Anteil an den Gesamtkosten ausmacht (Neunteufel et al., 2004).

Im gegenständlichen Projekt wurden die Prozesse Neuerrichtung von Haupt- und Versorgungsleitungen, die Rehabilitation von Haupt- und Versorgungsleitungen 
sowie die Erneuerung von Hausanschlussleitungen untersucht. Der Neubau von Hausanschlüssen wurde aufgrund zu geringer Teilnahmeanmeldungen zurückgestellt. Auch für die untersuchten Prozesse wurde erkannt, dass die verglichenen Leitungsbauprojekte entweder in ihrer Anzahl zu gering oder in ihrer Durchführung $\mathrm{zu}$ unterschiedlich waren, um die gewünschte Vergleichbarkeit bereits in diesem Pilotvorhaben herzustellen (siehe z. B. Tabelle 2). In Ergebnisanalyse-Workshops am 20./21.11.2007 in Linz konnten aber wichtige methodische Erkenntnisse zum ProzessBenchmarking im Themenbereich Leitungsbau abgeleitet werden. Beispielsweise mit der Vorgabe von enger umgrenzten „Standardprojekten“ (ähnlicher Durchmesser, verdichteter Siedlungsbereich, alle Prozessschritte erfüllt etc.) wurden konkrete Maßnahmen festgelegt, die zukünftige Vergleiche von Leitungsbauprojekten auf einem qualitativ hochwertigem Niveau ermöglichen sollen.

Weiters wurde in den Workshops ein intensiver Erfahrungsaustausch $\mathrm{zu}$ einzelnen Themenschwerpunkten gepflegt:

- Abhängigkeit der Preise für Erdarbeiten von der Wahl zwischen Jahresbauvertrag und Einzelausschreibungen?

- Beeinflussbarkeit der Laufmeterkosten und Vorgaben der Straßenverwaltungen? Regionale Marktunterschiede?

- Kostenteilung bei Mitverlegungen von/mit anderen Leitungsträgern? Kostenvorteile?

- Materialwahl und dessen Lebensdauer?

- Verfahrenswahl (Künette, Relining, Berstlining etc.)?

Werden die Prozessvergleiche mit größeren Stichproben und ähnlicheren Bauprojekten durchgeführt, können diese Fragen in Zukunft auch mit vergleichbaren Zahlen hinterlegt werden.

\subsection{Themenbereich Netzbetrieb und Instandhaltung}

In diesem Themenbereich wurden die Prozesse Wasserverlustmanagement und Netzinspektion angeboten. Der Großteil der Anmeldungen konzentrierte sich auf das Wasserverlustmanagement, weswegen dieser Prozess zur Durchführung gelangte und die Netzinspektion für einen späteren Zeitpunkt zurückgestellt wurde.

Beim Wasserverlustmanagement handelt es sich um einen komplexeren Prozess, der in viele Aufgabenbereiche eines Wasserversorgungsunternehmens eingreift. Die für diesen Prozess definierte Prozessstruktur umfasst somit nicht nur die vier Subprozesse

- Wasserverlustüberwachung,

- Leckortung,

- Reparatur sowie

- Analyse und Planung

zu denen Kosten und Arbeitszeiten erfasst werden, sondern zusätzlich auch noch die Hilfsprozesse Mitarbeiterqualifikation und Infrastrukturmanagement (z. B. Zählerwesen, Inspektion und Wartung, Rehabilitationsplanung etc.), die nur qualitativ über Kontextinformationen in die Prozessbewertung eingehen.

Wie in Abbildung 4 ersichtlich, nahmen 11 Wasserwerke unterschiedlichster Struktur am Projekt teil. Die Höhe der Wasserverluste ist innerhalb der strukturell vergleichbaren Gruppen (ländlich/ städtisch/großstädtisch) recht unterschiedlich. Bei der Berechnung der Wasserverluste wurden auch die Genauigkeiten der Eingangsdaten berücksichtigt. Die roten Linien in Abbildung 4 markieren die Grenzen der möglichen Schwankungsbereiche der jeweiligen Werte, basierend auf den erhobenen Messungenauigkeiten.

Nach den Richtwerten der DVGW W 392 (2003) in Tabelle 3 liegen drei der vier großstädtischen Wasserversorgungsunternehmen im Bereich hoher Wasserverluste
(WVU Nr. 2, 4 und 6) und ein großstädtisches Unternehmen im Bereich niedriger Verluste (Nr. 8). Bei den städtischen Teilnehmern liegt ein Wasserversorgungsunternehmen im Bereich „hoch“ (Nr. 7), zwei Unternehmen im Bereich mittlerer Verluste (Nr. 5 und 10) und ein Wasserversorgungsunternehmen im Bereich niedriger Verluste (Nr. 9). Alle drei ländlichen Wasserversorgungsunternehmen liegen nach dieser Klassifikation im Bereich mittlerer Verluste (Nr. 1, 3 und 11).

Die Bewertung der Gesamtkosten ist bei einem so umfangreichen Prozess und bei einem sehr heterogen strukturierten TeilnehmerInnenfeld durchaus kritisch zu betrachten. Beispielhaft zeigt Abbildung 5 die Kosten für den Subprozess Leckortung im Vergleich zum Anteil der jährlich mittels Leckortungsverfahren untersuchten Haupt- und Versorgungsleitungen. Dabei werden zwei Gruppen unterschieden: in blau Wasserversorgungsunternehmen mit anlassbezogener Leckortungsstrategie (d. h. es wird nur Leckortung betrieben, wenn es einen konkreten Verdacht aus der Wasserverlustüberwachung gibt) und in orange Wasserversorgungsunternehmen mit turnusmäßiger Strategie (d. h. über Leckortungskampagnen werden jährlich gewisse Anteile des Rohrnetzes inspiziert). Jene Unternehmen mit anlassbezogener Leckortungsstrategie untersuchen jährlich einen deutlich geringeren Anteil ihres Netzes und haben daher für die Leckortung auch einen deutlich geringeren Aufwand als die meisten Unternehmen mit turnusmäßiger Leckortungsstrategie, müssen aber natürlich höhere Aufwendungen im Subprozess der Wasserverlustüberwachung in Kauf nehmen.

Zur Beurteilung der Qualität der Prozessdurchführung wurde eine Qualitätsmatrix mit insgesamt ca. 100 Einzelabfragen zu den verschiedenen Subprozessen und Hilfsprozessen entwickelt. Jede dieser Ein- 


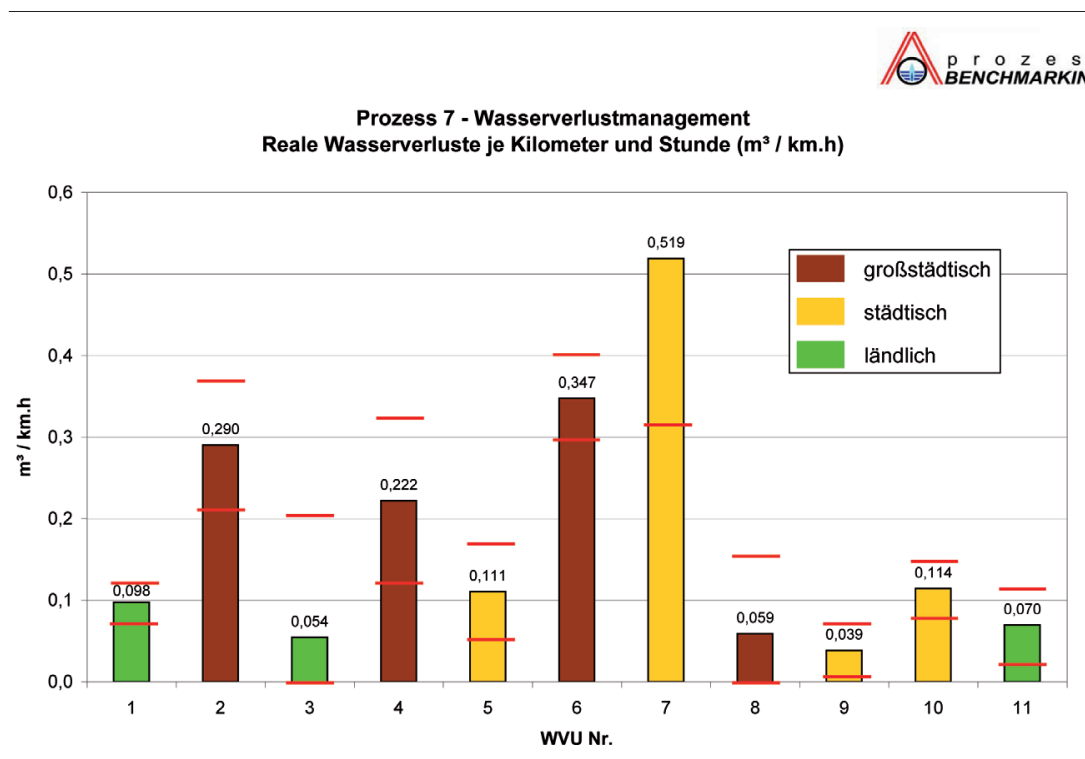

Abb. 4: Reale Verluste je Kilometer und Stunde (Datenbasis 2006), (rote Linien: min. / max.)

Abb. 5: Gesamtkosten für Leckortung im Vergleich zum Anteil an untersuchten Leitungen

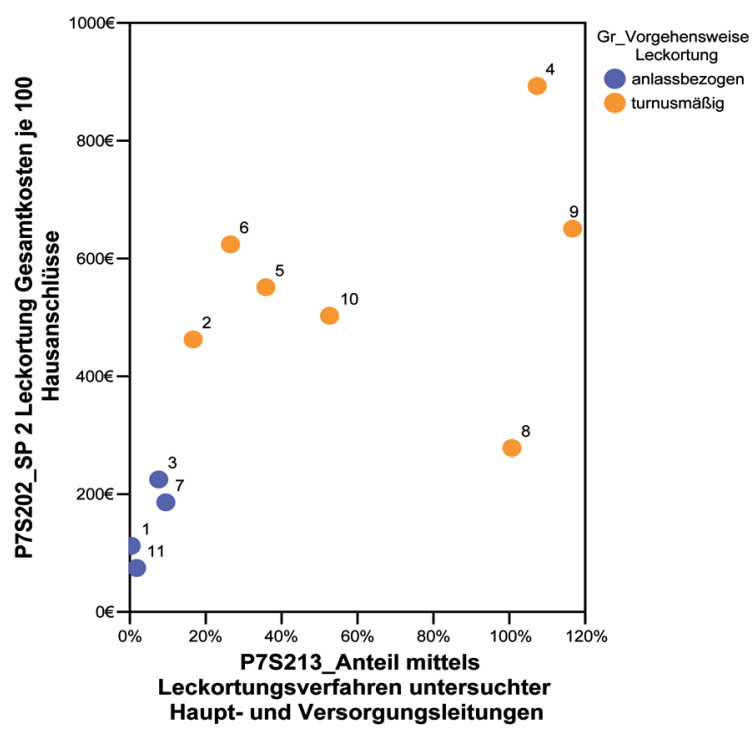

zelabfragen wird in der Matrix nach dem jeweiligen Qualitätsgrad auf einer 5-teiligen Skala färbig (rot... niedriger Qualitätsgrad bis grün... hoher Qualitätsgrad) dargestellt. Diese Qualitätsmatrix ermöglicht einen guten Überblick über die Besonderheiten und Optimierungspotentiale in qualitativer Hinsicht (Abbildung 6).

Das Prozess-Benchmarking zum Wasserverlustmanagement wurde wie die anderen Prozesse dieses Projektes in Österreich zum ersten Mal durchgeführt und stellt in dieser Form auch international eine Innovation dar. Mit Hilfe eines motivierten TeilnehmerInnenfeldes ist es gelungen, einen ersten Praxistest des entwickelten Systems erfolgreich $\mathrm{zu}$ absolvieren. Dem Feedback der teilnehmenden Wasserversorgungsunternehmen zufolge war das gegenständliche Projekt sogar mehr als nur ein Praxistest, und es ist gelungen, einen Vergleich der unterschiedlichen Vorgehenswesen im Wasserverlustmanagement für verschiedene Strukturen und Größen von Wasserversorgungsunternehmen anzustellen und darauf aufbauend Erkenntnisse für die Implementierung von Verbesserungsmaßnahmen abzuleiten.

\section{Zusammenfassung}

Ergänzend zum UnternehmensBenchmarking initiierten die

Tab. 3: Richtwerte für Reale Verluste pro Kilometer Leitungslänge nach DVGW W $392\left(\mathrm{~m}^{3} / \mathrm{km} . \mathrm{h}\right)$

\begin{tabular}{|c|c|c|c|}
\hline \multirow[b]{2}{*}{ Wasserverlustbereich } & \multicolumn{3}{|c|}{ Versorgungsstruktur } \\
\hline & $\begin{array}{c}\text { Bereich } 1 \text { (großstädtisch) } \\
\text { spez. Netzabgabe } \\
>15.000 \mathrm{~m}^{3} / \mathrm{km} . \mathrm{a}\end{array}$ & $\begin{array}{c}\text { Bereich } 2 \text { (städtisch) } \\
\text { spez. Netzabgabe } \\
5.000-15.000 \mathrm{~m}^{3} / \mathrm{km} . \mathrm{a}\end{array}$ & $\begin{array}{c}\text { Bereich } 3 \text { (ländlich) } \\
\text { spez. Netzabgabe } \\
<5.000 \mathrm{~m}^{3} / \mathrm{km} . \mathrm{a}\end{array}$ \\
\hline Geringe Wasserverluste & $<0,10$ & $<0,07$ & $<0,05$ \\
\hline Mittlere Wasserverluste & $0,10-0,20$ & $0,07-0,15$ & $0,05-0,10$ \\
\hline Hohe Wasserverluste & $>0,20$ & $>0,15$ & $>0,10$ \\
\hline
\end{tabular}


TU Graz und die BOKU Wien 2007 ein Projekt zum Prozess-Benchmarking in der österreichischen Trinkwasserversorgung, wobei unter Trägerschaft der ÖVGW und auf freiwilliger Basis ausgewählte Prozesse zu drei verschiedenen Themenbereichen untersucht wurden.

Obwohldie teilnehmenden Wasserversorgungsunternehmen beim Prozess-Benchmarking Neuland beschritten, waren die ersten Erfahrungen $\mathrm{zu}$ den Themenbereichen Wasserverkauf und Netzbetrieb und Instandhaltung großteils positiv. Beim Themenbereich Leitungsbau war aufgrund der geringen Teilnehmerzahl und der Heterogenität der untersuchten Bauprojekte die Vergleichbarkeit nicht in ausreichendem Maße gegeben. Dennoch konnten im TeilnehmerInnenkreis wichtige methodische Erkenntnisse erarbeitet werden, die ein künftiges Prozess-Benchmarking im Leitungsbau auf der Basis von Standardprojekten mit vergleichbaren Daten ermöglichen werden.

Die Gliederungen der verschiedenen Prozessstrukturen wurden von den teilnehmenden Wasserversorgungsunternehmen als gut und praxisnah bewertet. Es hat sich gezeigt, dass die Berechnung von Kennzahlen zu den Gesamtprozessen sowie zu den einzelnen Subprozessen und Varianten eine essentielle Basis für die Diskussion auf quantitativer Ebene darstellen, insbesondere deshalb, weil die

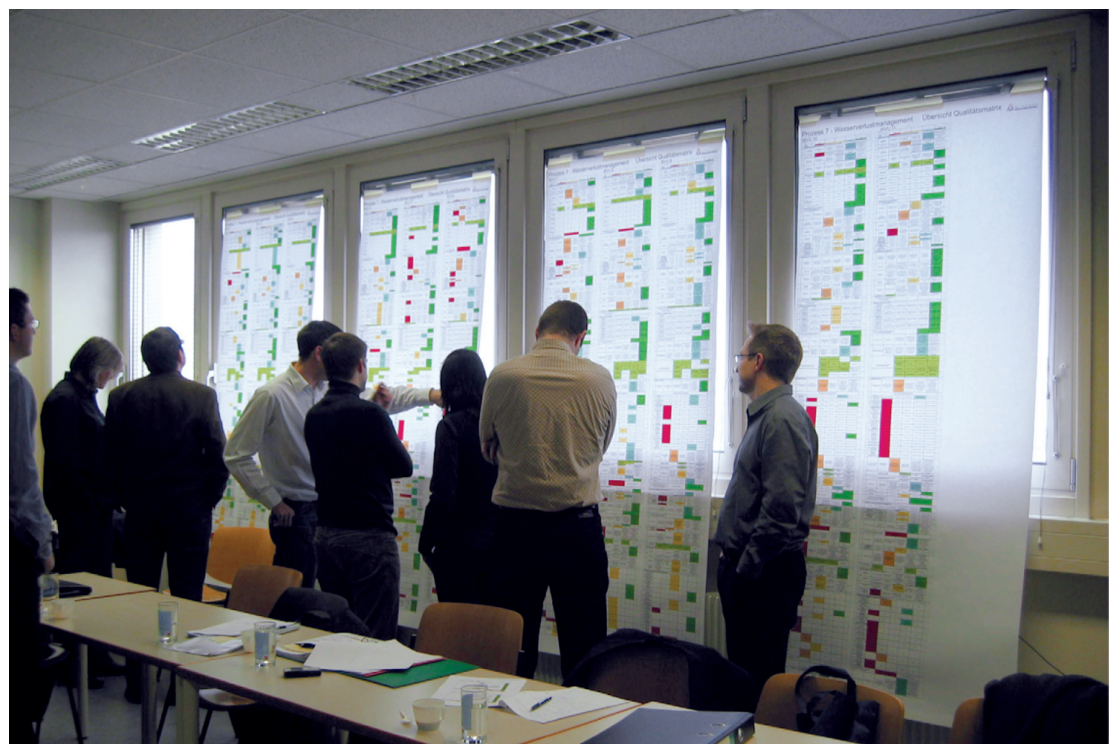

Abb. 6: Analyse der Qualitätsmatrix im Workshop

Vergleichbarkeit oft nur auf Ebene der Subprozesse oder Teilaufgaben erreicht werden kann. Ohne entsprechende Kennzahlen wäre das Erkennen von Effizienzsteigerungspotentialen nur schwer möglich.

Insgesamt hat sich die Kombination von wissenschaftlicher Methodik und prozessorientierter Anwendung bewährt, und es konnten sehr gute Ergebnisse in methodischer und fachlicher Sicht erzielt werden.

\section{Korrespondenz:}

${ }^{1}$ Technische Universität Graz, Institut für Siedlungswasserwirtschaft und Landschaftswasserbau,

Stremayrgasse 10/I, 8010 Graz

E-Mail: koelb|@sww.tugraz.at

${ }^{2}$ Universität für Bodenkultur, Institut für Siedlungswasserbau, Industriewasserwirtschaft und Gewässerschutz, Muthgasse 18, 1190 Wien

E-Mail: ernest.mayr@boku.ac.at

\section{LITERATUR}

DVGW - Arbeitsblatt W 392 (2003) Rohrnetzinspektion und Wasserverluste - Maßnahmen, Verfahren und Bewertungen. - DVGW, Bonn, Deutschland.

Kölbl, J., Theuretzbacher-Fritz, H., Neunteufel, R. Mayr, E. und Perfler, R. (2008) Benchmarking the Processes of Customer Meter Reading and Customer Meter Replacement - Performance Assessment of Urb Infrastructure Services. IWA Publishing Lnt of Urban Infrastructure Services. IWA Publishing, London, UK
ISBN 978-18-433-9191-3. Neunteufel, R., Theuretzbacher-Fritz, H., Teix, P. Kölbl, J. und Perfler, R. (2004) Benchmarking und Best Practices in der österreichischen Wasserversorgung Stufe A. - Endbericht des ÖVGW Pilotprojektes 2003/04, ÖVGW, Wien.

Ottilinger, F. (2004) EffWB-Untersuchung - Nutzen Ottilinger, F. (2004) EffWB-Untersuchung - Nutzen
der Untersuchung für die interne Kosten-/Leistungsrechnung und Steuerung. - Tagungsband Benchmarking Fachsymposium Effizienz- und Qualitätsuntersuchung der Kommunalen Wasserversorgung in Bayern (EffWB), Nürnberg, Deutschland. 